\title{
Tecnologías de impresión 3D: evaluaciones de FDM y Polyjet en la fabricación de autopartes de automóviles
}

\section{(3D printing technologies: FDM and Polyjet evaluations in the manufacture of automobile auto parts)}

\author{
Bryan Jhon Briceño-Martínez ${ }^{1,2}$, Edilberto Antonio Llanes-Cedeño, Juan Carlos Rocha-Hoyos ${ }^{1}$ \\ Edwin Chamba1 ${ }^{1}$, Diego Cuasapud ${ }^{1}$, Andrés Cárdenaz-Yánez ${ }^{13}$
}

\section{Resumen}

El objetivo del estudio fue evaluar las tecnologías de impresión 3D realizadas por Modelado Deposición Fundida (FDM) y PolyJet, a partir de ensayos mecánicos con materiales de Acrilonitrilo Estireno Acrilato (ASA) y VeroWhitePlus RGD 835 para la selección del material y tecnología adecuada en la fabricación de ductos de aire aplicados a autopartes automotrices. Con el fin de alcanzar el objetivo se realizaron pruebas de tracción mecánica a probetas fabricadas por ambas tecnologías; según la norma ASTM D638-14 para las pruebas de tracción y para los ensayos a flexión con la norma ASTM 790; para evaluar el comportamiento del flujo del aire en ducto se seleccionó como caso de estudio el ducto de aire empleado en la camioneta pick up Toyota Stout 2200 modelado por medio del software Ansys Student. Los resultados mostraron que el material VeroWhitePlus RDG 835 utilizado en la construcción del ducto por medio de la tecnología PolyJet presentó los mejores resultados con límite de fluencia a la tracción de 4.7 MPa y esfuerzo máximo de flexión de 28 MPa.

\section{Palabras clave}

Autopartes; FDM; PolyJet; Impresión en 3D; ensayos mecánicos.

\begin{abstract}
The aim of the study was to evaluate the 3D printing technologies made by Molded Deposition Modeling (FDM) and PolyJet from mechanical tests with Acrylonitrile Styrene Acrylate (ASA) and VeroWhitePlus RGD 835 materials, for the selection of the material and adequate technology in the manufacture of air ducts applied to automotive auto parts. To achieve theis purpose, mechanical tensile tests were carried out on specimens manufactured by both technologies, according to ASTM D638-14 for tensile tests and for bending tests according to ASTM 790. To evaluate the behavior of air flow in the pipeline, the air duct used in the Toyota Stout 2200 pick-up truck modeled by the Ansys Student software was selected. The results showed that the VeroWhitePlus RDG 835 material used in the construction of the duct by means of the PolyJet technology presented the best results with a yield strength of $4.7 \mathrm{MPa}$ and a maximum bending stress of $28 \mathrm{MPa}$.
\end{abstract}

\section{Keywords}

Auto parts; FDM; PolyJet; 3D printing; mechanical tests.

\section{Introducción}

La ASTM (2012) describe el proceso de diseño asistido por computadora impresión 3D, como el proceso de materializar diseños gráficos tridimensionales digitales en un objeto físico por la sobreposición de capas de material, utilizando un cabezal de impresión, boquilla u otra tecnología de la impresora. Esta tecnología fue patentada por Charles Hulk en 1984, con el nombre de STereoLithography denominado prototipado rápido, siendo una realidad comercial en 1987,

\footnotetext{
1 Universidad Internacional SEK, Quito, Ecuador (bbriceno.mdmauisek.edu.ec, antonio.llanes@uisek.edu.ec, carlos.rocha@ uisek.edu.ec, echamba.mdm@uisek.edu.ec, dcuasapud.mdm@uisek.edu.ec, rcardenas.mdm@uisek.edu.ec).

2 Universidad Nacional de Loja, Loja, Ecuador (bryan.briceno@unl.edu.ec).

3 Ministerio de Salud Pública, Quito, Ecuador (acardenasyanez@hotmail.com).
} 
con 3D Systems. En 1990 se empieza a aplicar la fabricación aditiva (AM), en contraposición a la tecnología de sustracción como el mecanizado metalmecánico, utilizado en tecnologías de producción y en cadenas de suministro, para obtener patrones de fundición. En 1995, fue utilizada la AM para obtener herramientas de producción, especialmente moldes de inyección en 2000. En adelante, las patentes de estereolitografía y de modelado por deposición fundida (FDM) ha dado lugar a la aparición de las impresoras personales, basadas en "fundir" hilo de plástico (Villagomez, Caracheo, Mondragón, y Castro, 2017).

La expansión y disminución de precios en el mercado de impresoras 3D fue el factor económico en la evolución del conocimiento asociado a esta tecnología y a los nuevos materiales, la cual permite masificar el sistema productivo y un cambio en los paradigmas de producción (Soto de Vicente, 2014). Entre las características positivas del uso de la impresión 3D, se destaca la fabricación rápida de prototipos 3D, la reducción del stock de piezas poco comunes en almacenes de autopartes, la producción de piezas que requieren customización como prótesis y venta de objetos con poca demanda que no compensa hacer un lote previo de unidades (Municipalidad de Cordoba, 2014).

La tecnología FDM trabaja con impresoras 3D, aplicadas a termoplásticos con el fin de construir piezas resistentes, duraderas y dimensionalmente estables, con buena precisión y repetibilidad de cualquier tecnología de impresión 3D; algunos beneficios de FDM son: su tecnología limpia, fácil de usar y amigablemente estables, resolviendo geometrías complejas de construcción. PolyJet es una potente tecnología de impresión en 3D que produce piezas, prototipos y herramientas de acabado suave y de gran precisión, aportando una resolución de capa microscópica y precisión de hasta de $0.01 \mathrm{~mm}$, además produce paredes finas y geometrías complejas, aportando una gama amplia de materiales, algunas ventajas de PolyJet es generar moldes, guías y fijaciones, incorporando mayor variedad de colores.

En el campo de la automoción se continúa investigando acerca de materiales livianos y resistentes, que pueden producirse a través de impresiones 3D. La fabricación de ductos de aire, alerones, accesorios plásticos son entre otros los usos más frecuentes que se están desarrollando. Las industrias y talleres, no necesitan solicitar al proveedor los repuestos de un automóvil cuando se produce una falla, gracias a la impresora 3D se puede producir una réplica del o los repuestos, y con este medio de impresión se puede resolver la falla presentada en los vehículos.

El presente artículo, se basa en una evaluación de manufactura de las tecnologías de impresión 3D, modelado por deposición fundida (FDM) y fabricación aditiva (PolyJet), a partir de comparaciones, estudios computacionales de modelación y fabricación de probetas con dichas tecnologías, utilizando como material constructivo al Acrilonitrilo Estireno Acrilato (ASA) y VeroWhitePlus RGD83 para la aplicación en la fabricación de un conducto de aire automotriz.

\section{Materiales y métodos}

Para alcanzar el objetivo de la investigación se ejecutaron un conjunto de pasos: a) selección de materiales, b) caracterización c) diseño CAD, d) análisis CAE: CFD y estructural, e) selección del material apropiado, y f) construcción por AM (Vega et al., 2018).

\section{Selección de materiales}

Para utilizar la tecnología FDM se preseleccionaron tres materiales: Acrilonitrilo Estireno Acrilato (ASA), Acrilonitrilo Butadieno Estireno (ABSi) y FDM Nylon 6; de forma equivalente para la 
tecnología PolyJet se utilizaron los fotopolímeros: VeroWhitePlus RGD835, VeroGlaze MED620 y TangoBlack FLX973. Basándose en un estudio comparativo de materiales existentes en el mercado ecuatoriano (Briceño Martínez, 2018) y en las propiedades de materiales ofrecidas por Stratasys (2018) como son: rendimiento, color, durabilidad, rigidez, resistencia a los rayos UV; se selecciona el material Acrilonitrilo Estireno Acrilato (ASA) para uso en FDM y el VeroWhitePlus RGD835 para uso con PolyJet.

\section{Caracterización de materiales}

Con el fin de conocer las propiedades mecánicas: límite de fluencia $\left(S_{\gamma}\right)$, resistencia última $\left(S_{u}\right)$, módulo de flexión $\left(E_{f}\right)$ y módulo elástico $(E)$, necesarias para los análisis en CAE, se realizan ensayos de tracción y flexión para plásticos rígidos y semi-rígidos según lo referenciado por Cazón y Morer (2014), Goncalves (2002) y Paredes, Pérez, Miniguano, y Christian (2017). Análisis de las propiedades mecánicas del compuesto de matriz poliéster reforzado con fibra de vidrio 375 y cabuya aplicado a la industria automotriz. Enfoque UTE, 8 (3): 1-15. El ensayo de tracción está basado en la norma ASTM D638-14 (ASTM International, 2014), la muestra a elegir es el Tipo I, utilizado cuando se dispone de material suficiente con un espesor de $7 \mathrm{~mm}$ o menos. El espesor del modelo Tipo I será $3.2 \pm 4$ mm. La dimensión de la probeta de tracción Tipo I se muestra en la Figura 1. El número de probetas a ensayar que recomienda la norma son mínimo de cinco. La velocidad de prueba del ensayo es de $5 \pm 25 \% \mathrm{~mm} / \mathrm{min}$, la velocidad de deformación nominal al comienzo de la prueba es de $0.1 \mathrm{~mm} / \mathrm{min}$, el valor que alcanza la ruptura es en un tiempo de prueba de 0.5 a 5 minutos.

Los materiales utilizados para los ensayos de tracción y de flexión son: filamento de AcriIonitrilo Estireno Acrilato (ASA), del cual se fabrican 5 probetas aplicadas a la tecnología de Modelado de Fusión por Deposición (FDM) y de VeroWhitePlus RGD835, 5 probetas aplicadas a la tecnología PolyJet.

En el ensayo de flexión, la norma a utilizar es la ASTM 790-02 (ASTM International, 2002), la prueba es de tres puntos, aplicándose a un total de 10 probetas, 5 de filamento ASA, con una densidad de $1.07 \mathrm{~g} / \mathrm{cm}^{3}$, y 5 de VeroWhitePlus RGD835, con una densidad de 1,18 g/ $/ \mathrm{cm}^{3}$. Las dimensiones de las probetas de flexión se indican en la Figura 2.

Figura 1. Probeta utilizada en el ensayo de tracción (dimensiones en mm)

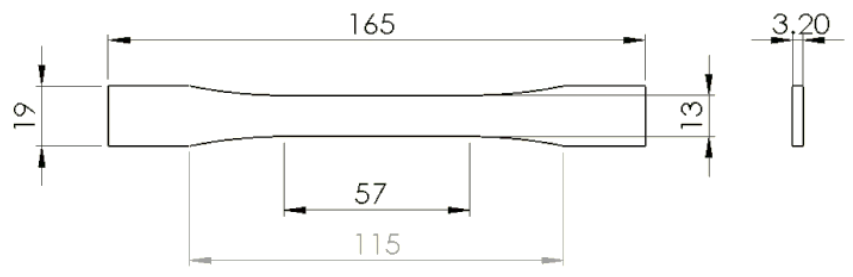

Figura 2. Probeta utilizada en el ensayo de flexión (dimensiones en mm)

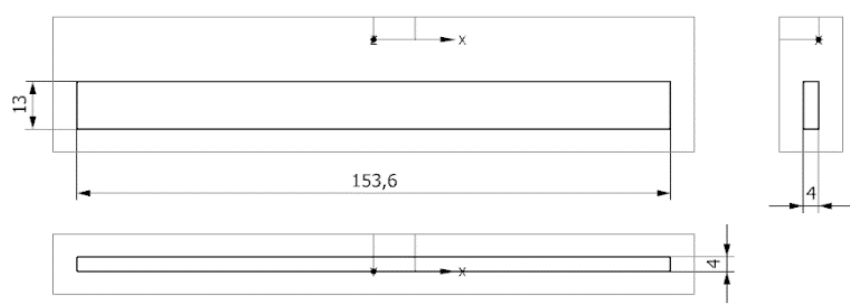


Las probetas son impresas en 3D para los ensayos de tracción (Figura 3) y ensayos de flexión (Figura 4), iguales en los dos materiales a ensayar.

Figura 3. Probetas impresas en 3D para ensayos a tracción

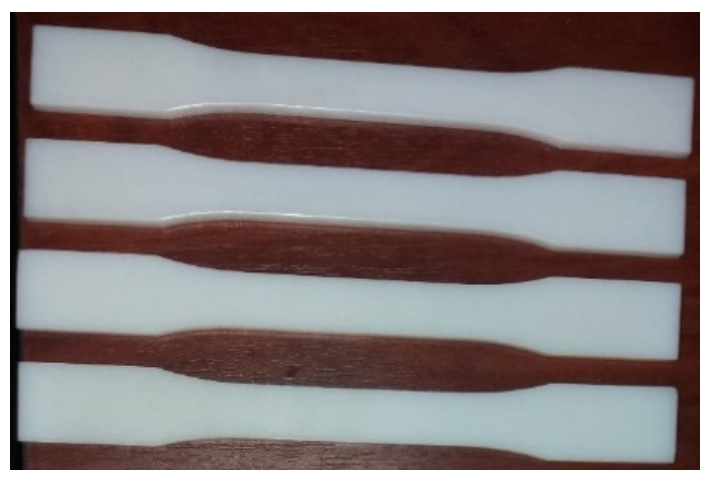

Figura 4. Probetas impresas en 3D para ensayos a flexión

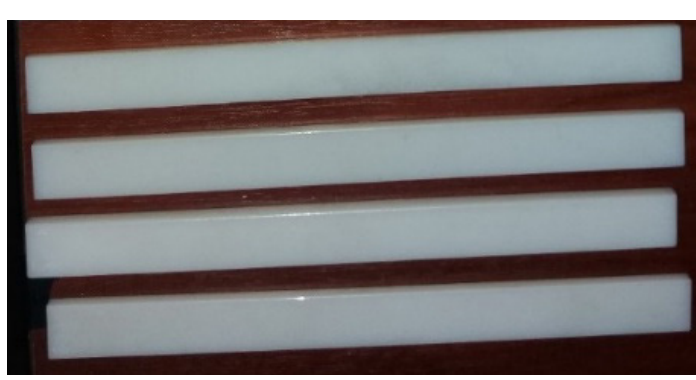

Los ensayos se efectúan en una máquina universal de ensayos Metrotec Serie MTE50, de 50 kN (Figura 5), del Laboratorio de Resistencia de Materiales del Centro de Fomento Productivo Metal Mecánico Carrocero, de Tungurahua, Ecuador, la misma es utilizada para experimentar con materiales poliméricos, cerámicos, compuestos y metales ligeros.

Figura 5. Máquina universal de ensayos Metrotec 50 kN

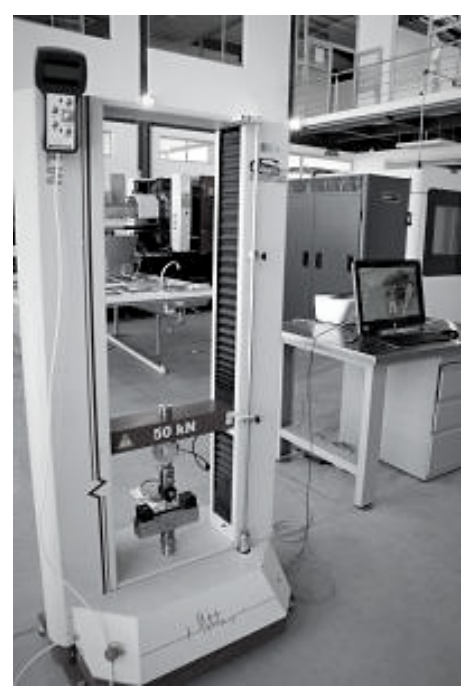




\section{Diseño CAD del ducto de aire}

La parte automotriz escogida para la experimentación es el ducto de aire de la camioneta Toyota Stout 2200. A partir de lo referido por López (2017) se aplica la ingeniería inversa mediante la medición de sus dimensiones geométricas para el diseño del modelo CAD por medio de la herramienta Siemens NX 11; en la Figura 6 se muestra la autoparte referida.

Figura 6. Ducto de Aire, Camioneta Toyota Stout 2200

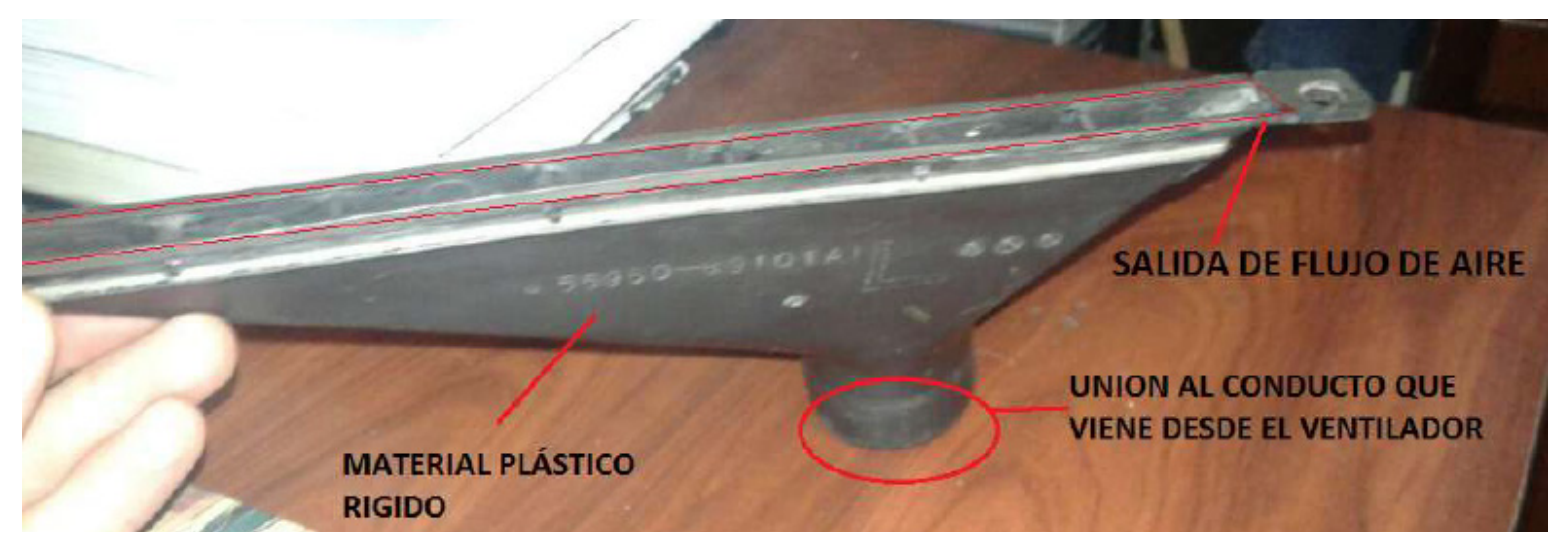

\section{Análisis CAE. Ensayos CFD}

A partir del modelo CAD se efectuo el análisis CFD por medio de los resultados de la simulación se observan los comportamientos de la presión, velocidad y puntos críticos del flujo en la geometría del conducto. Se genera la malla del volumen de control y se cargan las condiciones de frontera. El estudio se basa en un análisis de turbulencia k- $\varepsilon$ (k: energía cinética de turbulencia, $\varepsilon$ : velocidad de disipación de la energía de turbulencia), resuelto por diferencias finitas y simulado con FLUENT (Toapanta, Bohórquez, Caiza, y Sarzosa, 2018).

\section{Ensayos estructurales}

El análisis estructural, se realiza con el software Ansys Student. Generado el ducto de aire y su toma en CAD, se procede a exportar el ducto en formato tipo STEP para posteriormente importarlo a SPACECLAIM. Capturado el modelo, se procede a la extracción del volumen, se ingresa la geometría y se malla con un jacobino de 1, después se exporta la malla y se selecciona ANSYS CFD, se indican las condiciones de borde con velocidades de ingreso de $1.5 \mathrm{~m} / \mathrm{s}$, escogiéndose el modelo matemático a utilizar en este caso, turbulencia $\mathrm{k}-\varepsilon$, y por último se procesan los datos, verificando la convergencia (Lozada, y Suquillo, 2018).

\section{Selección de material constructivo}

De acuerdo a los resultados de las simulaciones, se escoge el material y tecnología a usar en la construcción de la autoparte. 


\section{Construcción de autoparte-ducto de aire}

Según el material escogido se procede a la construcción con tecnología AM, del modelo de autoparte-ducto de aire.

\section{Resultados}

En el siguiente epígrafe se detallan los resultados obtenidos al aplicar la impresión 3D en autopartes vehiculares (caso de estudio ducto de aire).

\section{Diseño CAD}

En la Figura 7, se muestra el conducto de aire virtual realizado usando software Siemens NX 11, el cual representa la primera etapa para su evaluación en software de simulación con CFD.

Figura 7. Conducto de aire diseñado, software Siemens Nx11.0

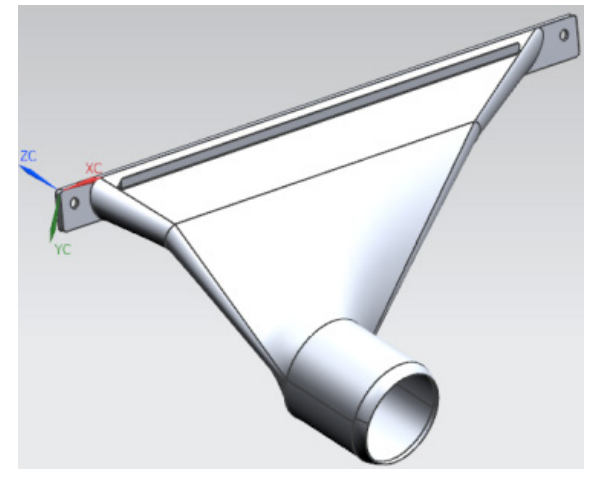

En la Figura 8 a, se visualiza el conducto flexible que va acoplado al ducto principal, el cual es ensamblado para posteriormente realizar un análisis CFD y estructural. El ensamble se indica en diferentes vistas en la Figura 8 b.

Figura 8. Vistas de ensamble del modelo a) conducto flexible b) ensamble

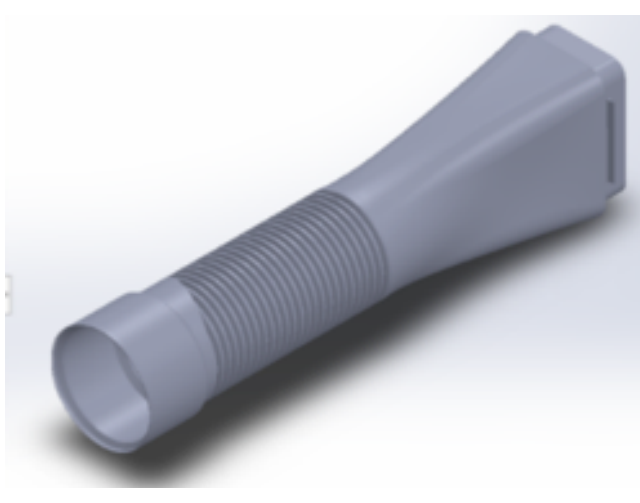

a)

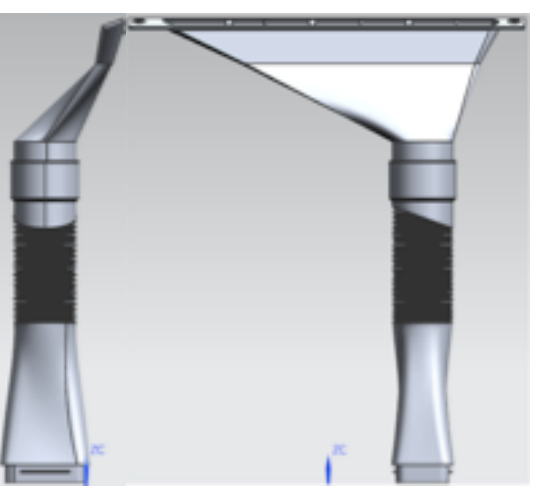

b) 


\section{Ensayo de tracción}

En la Figura 9 se muestra la máquina empleada para el ensayo de tracción con sus características.

Figura 9. Disposición para ensayo tracción

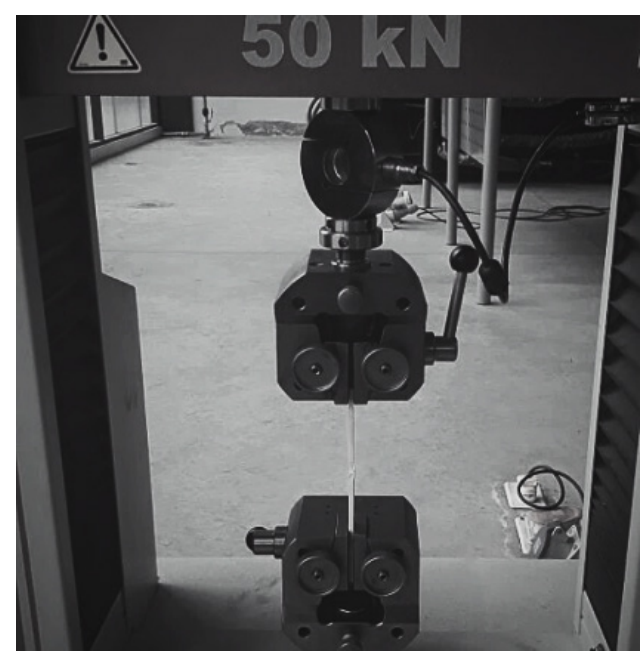

En la Figura 10 se presenta el ensayo de tracción del material VeroWhitePlus RGD835, en coordenadas de carga vs desplazamiento, y en la Tabla 1 se relacionan los parámetros principales para las 5 probetas experimentadas, relacionados con: la resistencia máxima $\left(S_{U}\right)$, el límite de fluencia $\left(S_{\gamma}\right)$, y el límite de rotura $\left(S_{R}\right)$.

Figura 10. Resultados Gráficos del ensayo de tracción, material VeroWhitePlus RGD835

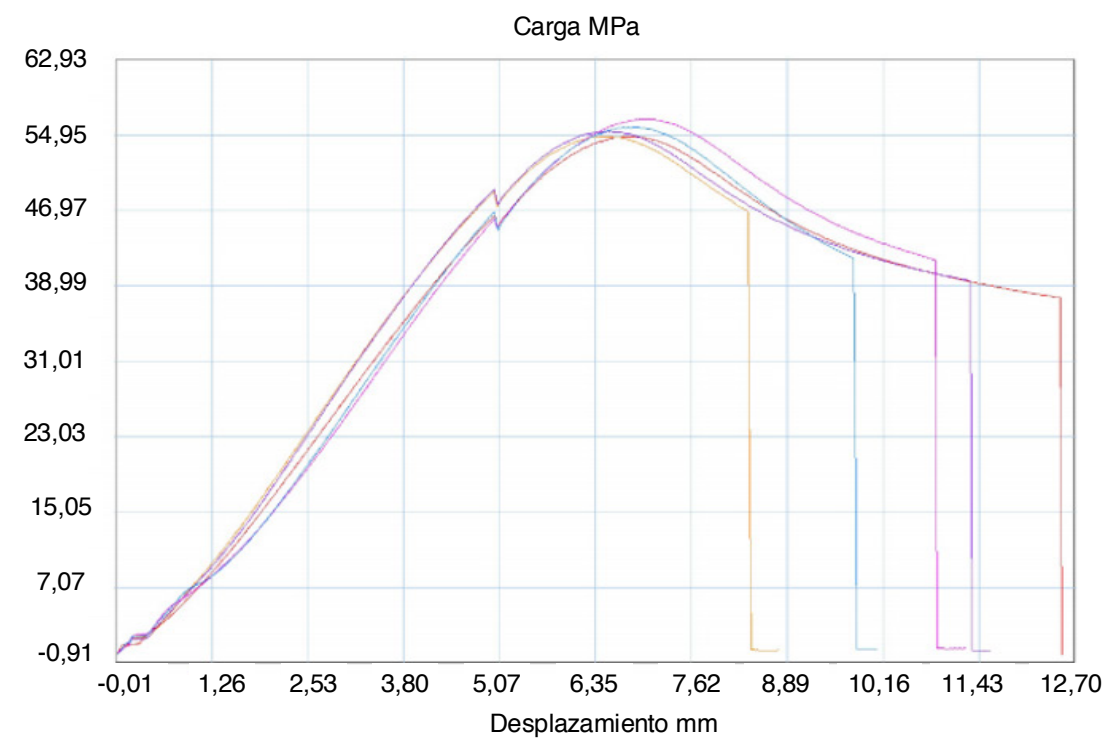


Tabla 1. Resistencia máxima $S_{U^{\prime}}$ límite de fluencia $S_{Y^{\prime}}$ límite de rotura $S_{R}-W V P R G D 835$.

\begin{tabular}{|l|c|c|c|}
\hline \multicolumn{1}{|c|}{ Probeta } & $\mathrm{S}_{\mathbf{U}}(\mathrm{MPa})$ & $\mathrm{S}_{\mathbf{Y}}(\mathrm{MPa})$ & $\mathrm{S}_{\mathrm{R}}(\mathrm{MPa})$ \\
\hline 1 & 54.79 & 4.61 & 37.72 \\
\hline 2 & 54.79 & 3.91 & 46.82 \\
\hline 3 & 55.34 & 4.90 & 39.49 \\
\hline 4 & 56.61 & 5.27 & 41.70 \\
\hline 5 & 55.78 & 4.81 & 41.85 \\
\hline Promedio & 55.46 & 4.70 & 41,52 \\
\hline
\end{tabular}

El resultado gráfico del ensayo de tracción para el material ASA, se muestra en la Figura $11, y$ en la Tabla 2 se indica $S_{U^{\prime}} S_{Y^{\prime}} S_{R}$ y el promedio para las 5 probetas experimentadas.

Figura 11. Resultados Gráficos del ensayo de tracción, material ASA

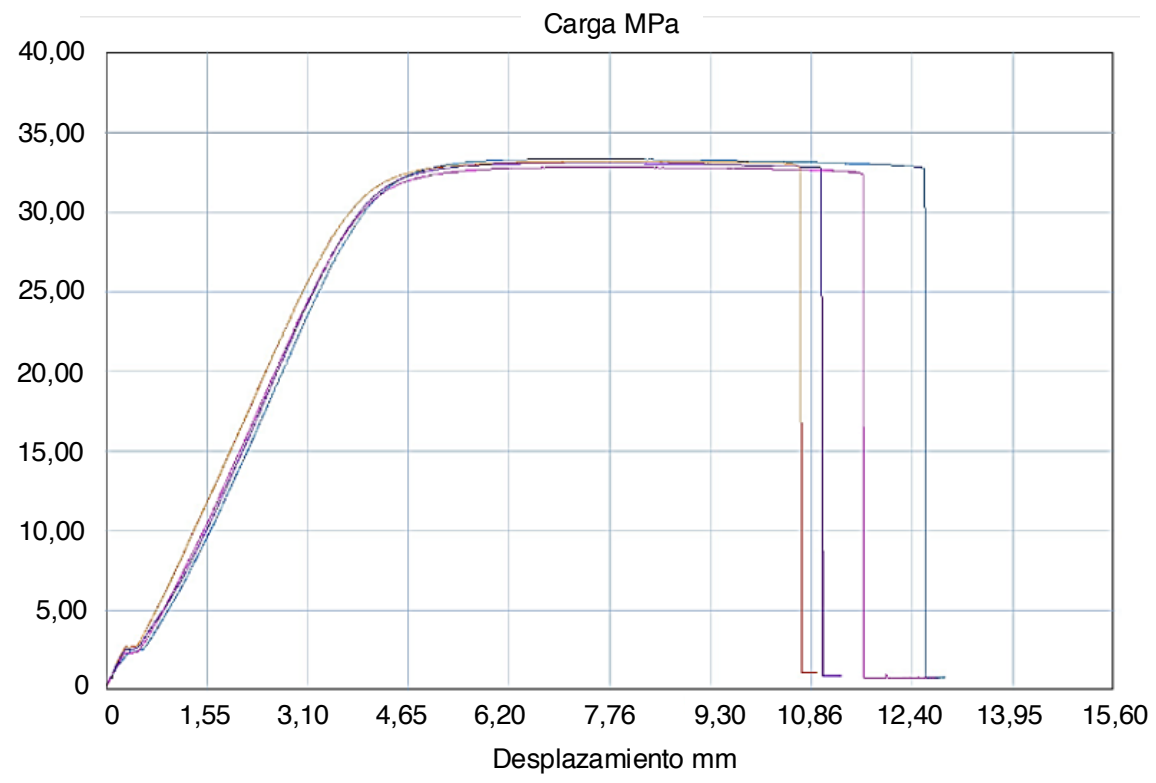

Tabla 2. Resistencia máxima $S_{U^{\prime}}$ límite de fluencia $S_{Y^{\prime}}$ Límite de rotura $S_{R}-A S A$.

\begin{tabular}{|l|l|l|l|}
\hline \multicolumn{1}{|c|}{ Probeta } & \multicolumn{1}{|c|}{$\mathrm{S}_{\mathrm{U}}(\mathrm{MPa})$} & \multicolumn{1}{c|}{$\mathrm{S}_{\mathrm{Y}}(\mathrm{MPa})$} & \multicolumn{2}{c|}{$\mathrm{S}_{\mathrm{R}}(\mathrm{MPa}$} \\
\hline 1 & No registra valores & 33.58 \\
\hline 2 & 34.24 & 2.7 & 33.47 \\
\hline 3 & 34,06 & 3.24 & 33.65 \\
\hline 4 & 33.97 & 3.03 & 33.13 \\
\hline 5 & 33.7 & 3.05 & 33.46 \\
\hline Promedio & 33.99 & 3.01 & \\
\hline
\end{tabular}




\section{Ensayo de flexión}

En laFigura 12 se muestra la máquina empleada para el ensayo de a flexión con sus características.

Figura 12. Disposición Metrotec Serie MTE50 para ensayo de flexión

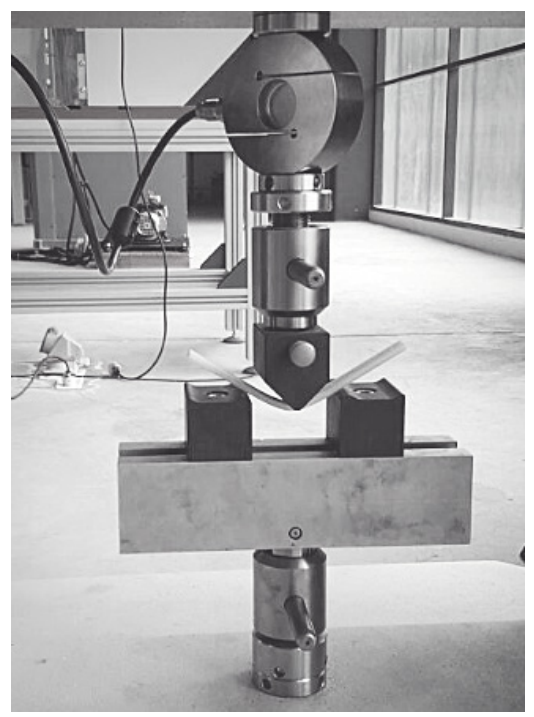

El resultado gráfico del ensayo de flexión aplicado a las probetas VWP RGD835 se indica en la Figura 13.

Figura 13. Resultados Gráficos del ensayo de flexión sometidos a fuerza, material VeroWhitePlus RGD835

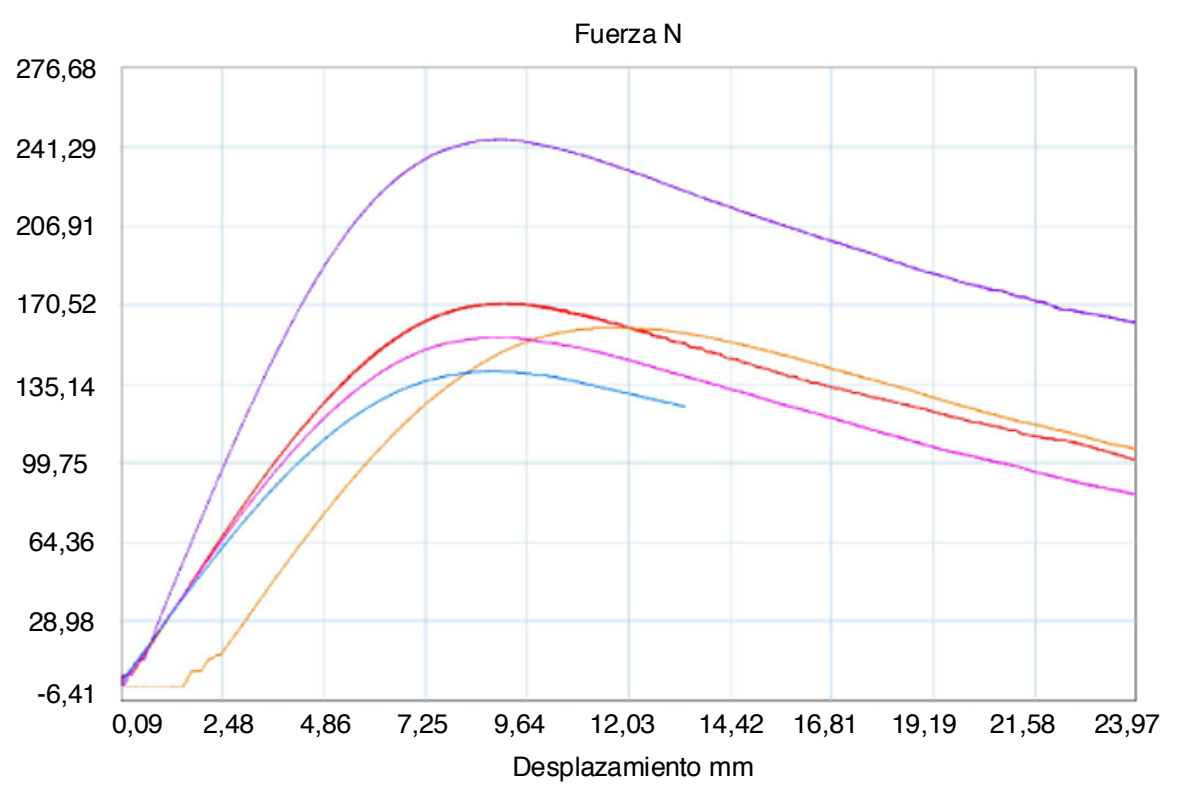

El esfuerzo máximo de flexión $\left(\sigma_{\text {fmax }}\right)$ y el módulo de elasticidad secante de flexión $\left(E_{f}\right)$, resultado de los ensayos de flexión aplicados a las probetas de material VWP RGD835, se muestran en la Tabla 3. 
Tabla 3. Valores de $\sigma_{f m a x}$ y $E_{f}$ del material VWP RGD835.

\begin{tabular}{|l|c|c|}
\hline \multicolumn{1}{|c|}{ Probeta } & \multicolumn{1}{|c|}{$\sigma_{\text {fmax }}(\mathrm{MPa})$} & $\mathrm{E}_{\mathrm{f}}(\mathrm{MPa})$ \\
\hline 1 & No registra valores & 166.33 \\
\hline 2 & 29.20 & 125.97 \\
\hline 3 & 23.85 & 124.9 \\
\hline 4 & 21.96 & 372.51 \\
\hline 5 & 36.89 & 197.43 \\
\hline Promedio & 27.98 & \\
\hline
\end{tabular}

La Figura 14, muestra el comportamiento del ensayo de flexión para las probetas ASA.

Figura 14. Resultados Gráficos del ensayo de flexión, material ASA

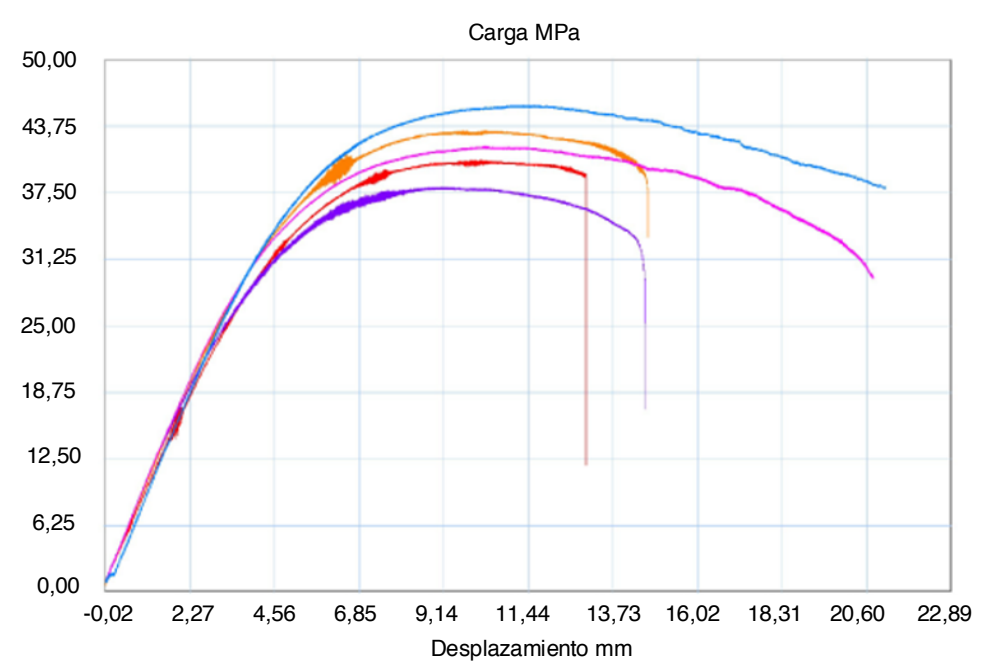

Los resultados de los ensayos de flexión para las probetas ASA, se indican en la Tabla 4.

Tabla 4. Valores $\sigma_{\text {fmax }}$ y Ef del material ASA

\begin{tabular}{|l|c|c|}
\hline \multicolumn{1}{|c|}{ Probeta } & $\sigma_{\text {fmax }}(\mathbf{M P a})$ & $\mathbf{E}_{\mathrm{f}}(\mathbf{M P a})$ \\
\hline 1 & 42.79 & 612.22 \\
\hline 2 & 44.88 & 590.61 \\
\hline 3 & 37.65 & 499.76 \\
\hline 4 & 43.00 & 499.94 \\
\hline 5 & 46.76 & 506.94 \\
\hline Promedio & 43.02 & 541.89 \\
\hline
\end{tabular}




\section{Análisis CFD}

En el estudio CFD se procede a calcular la presión total del ducto de aire dando como resultado 3.18 Pa, al ingreso de la toma del conducto (Figura 15).

Figura 15. Presión de la toma del ducto

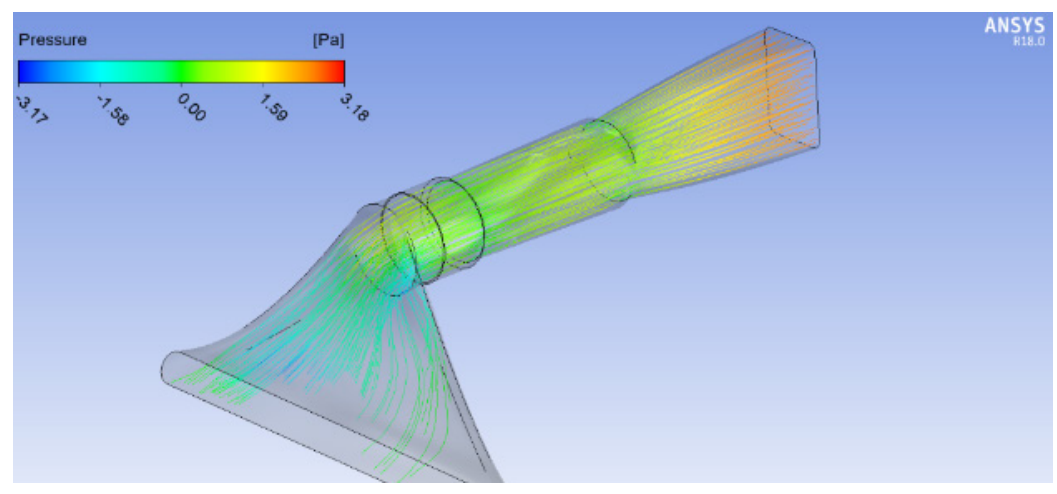

Las velocidades del flujo se determinan en los 3 ejes de coordenadas, mostrándose los siguientes valores: velocidades en la toma del ducto $u=2,30 \mathrm{~m} / \mathrm{s}$ (eje $\mathrm{x}$ ), $v=0.38 \mathrm{~m} / \mathrm{s}$ (eje y) y $\mathrm{w}=2.80 \mathrm{~m} / \mathrm{s}$ (eje z), como se indica en la Figura 16 .

Figure 16. Velocidades en ducto de aire, a) $u, b) \vee c) w$

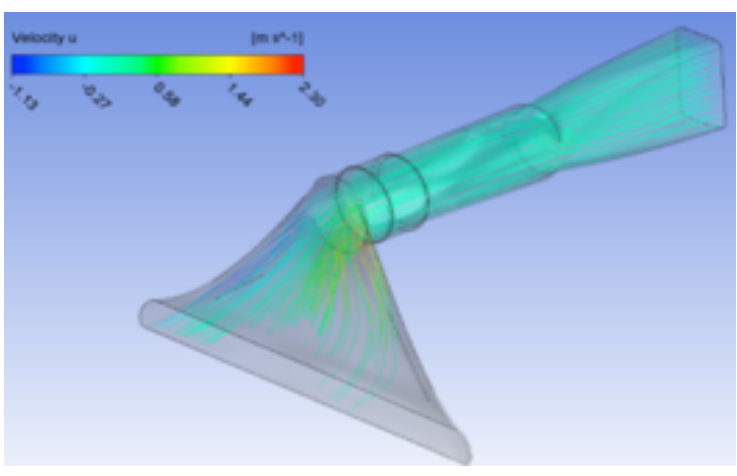

a)

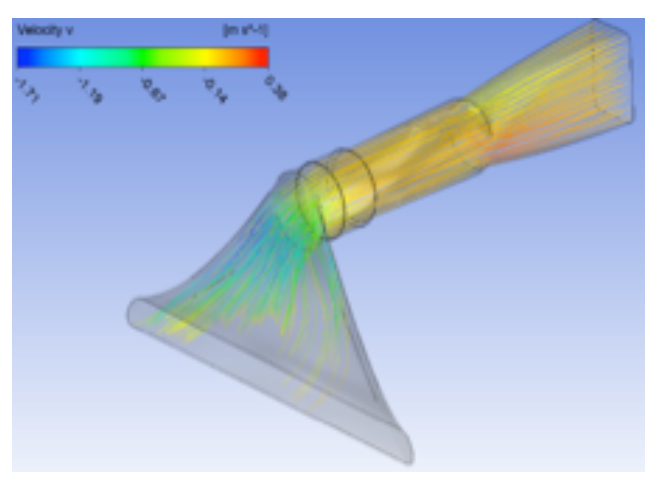

b)

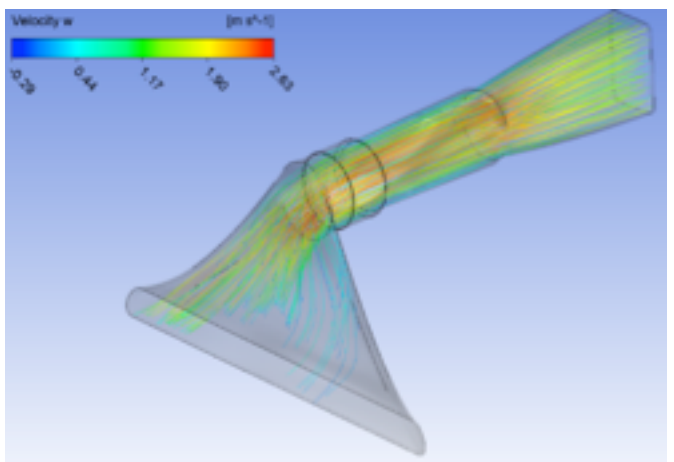

C) 
Comparación de materiales mediante Análisis Estructural (Polipropileno, ASA y VeroWhitePlus RGD835)

En la Tabla 5, se indican las propiedades mecánicas de los materiales analizados, polipropileno (escogido para efectos comparativos, por ser el material original de la pieza a construir), el ASA y el VeroWhitePlus RGD835.

Tabla 5. Propiedades mecánicas de materiales en simulación

\begin{tabular}{|l|c|c|c|}
\hline & VWP RGD835 & ASA & Polipropileno* \\
\hline$E(\mathrm{MPa})$ & 3000,00 & 541.89 & 1000 \\
\hline$\rho\left(\mathrm{g} / \mathrm{cm}^{3}\right)$ & 1.18 & 1.07 & 0.90 \\
\hline $\boldsymbol{E}$ & 0.33 & 0.33 & 0.40 \\
\hline$E S(\mathrm{MPa})$ & 197.43 & 541.89 & 1500 \\
\hline$S_{Y}(\mathrm{MPa})$ & 4.70 & 3.01 & 24 \\
\hline $\mathrm{S}_{U}(\mathrm{MPa})$ & 41.52 & 33,46 & 35 \\
\hline
\end{tabular}

Nota: *Propiedades tomadas de GIMDEPPM (2018).

En la Figura 17, 18 y 19 se encuentran los trazados de deformación total (u) y la intensidad de esfuerzo de von Mises $\left(\sigma_{\mathrm{vM}}\right)$ para el material VeroWhitePlus RGD835, ASA y polipropileno respectivamente.

Figura 17. Valores de: a) $u(\mathrm{~mm})$ y b) $\sigma_{\mathrm{vM}}(\mathrm{MPa})$ del material VWP RGD835

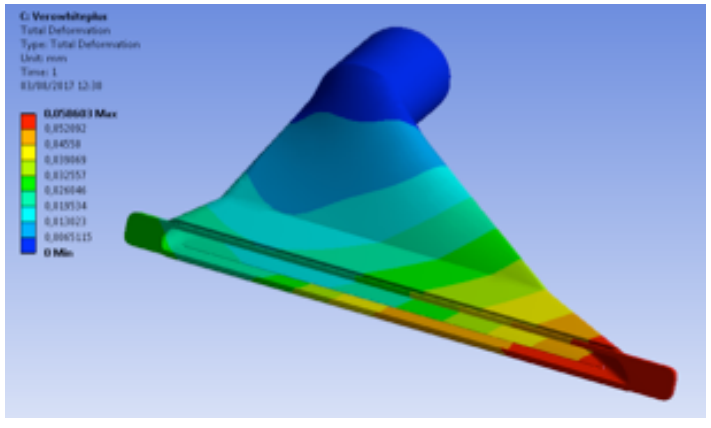

a)

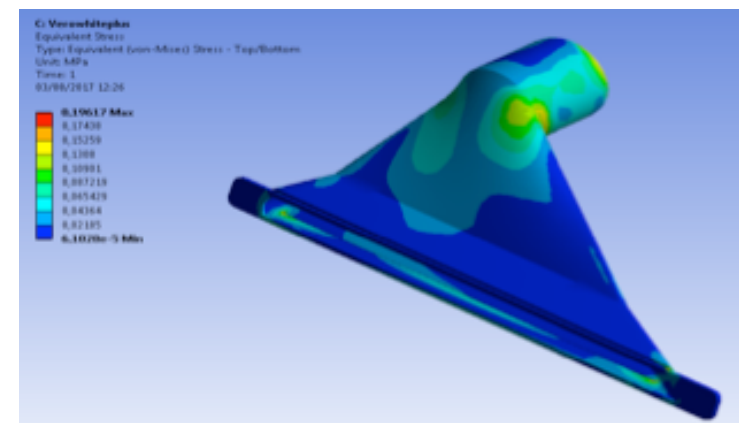

b)

Figura 18. Valores de: a) $u(\mathrm{~mm})$ y b) $\sigma_{\mathrm{vM}}(\mathrm{MPa})$ del material ASA

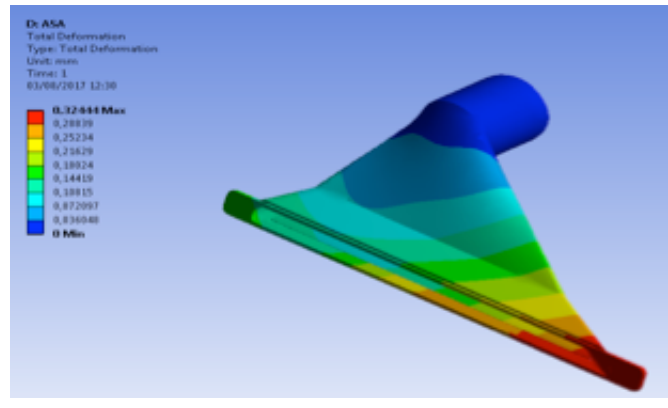

a)

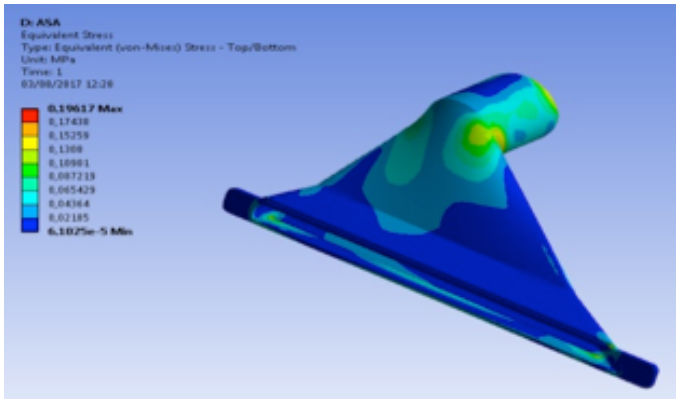

b) 
Figura 19. Valores de: a) $u(\mathrm{~mm})$ y b) $\sigma_{V M}(\mathrm{MPa})$ del material polipropileno

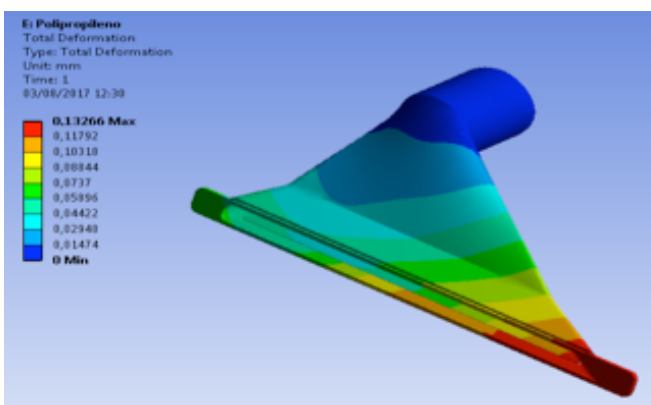

a)

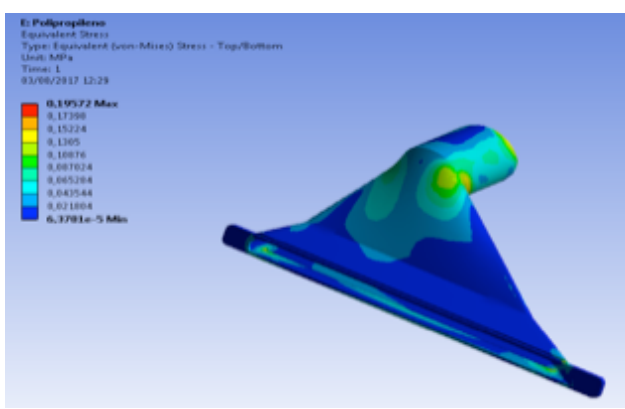

b)

La Tabla 6 , resume la deformación total (u) y la intensidad de esfuerzo de von Mises $\left(\sigma_{\mathrm{vM}}\right)$, para los tres materiales.

Tabla 6. Resultados de u $(\mathrm{mm})$ y $\sigma_{\mathrm{vM}}(\mathrm{MPa})$.

\begin{tabular}{|l|c|c|}
\hline & $\mathrm{u}(\mathrm{mm})$ & $\mathrm{vm}(\mathrm{MPa})$ \\
\hline VWP RGD835 & 0.05860 & 0.19617 \\
\hline ASA & 0.32444 & 0.19617 \\
\hline Polipropileno & 0.13266 & 0.19572 \\
\hline
\end{tabular}

\section{Fabricación del ducto de aire automotriz}

En la manufactura del ducto de aire se llevaron a cabo tres etapas de fabricación, la primera es el modelado por medio del software de impresión (Figura 20), el segundo es el conducto de aire ya impreso por partes (el motivo de las partes se debe a que por las dimensiones de la geometría no se acoplaron en la bandeja de impresión) (Figura 21), la siguiente etapa es unir con pegamento ultra fuerte y material de relleno VeroWhitePlus RGD835; ya unida y pegada se atraviesa una luz ultravioleta la cual se encargará de solidificar la pieza para su uso final (Figura 22).

La impresora en 3D utilizada es la Objet 30Pro (Figura 23); esta impresora pertenece a la tecnología PolyJet, la misma posee resinas acrílicas foto curadas con proceso de luz ultravioleta, destacando versatilidad en la impresión de materiales sustitutivos al polipropileno, el espacio de impresión es de $29.4 \mathrm{~cm} \times 19.2 \mathrm{~cm} \times 14.8 \mathrm{~cm}$.

Figura 20. Modelado del ducto en el software de la Impresora en 3D

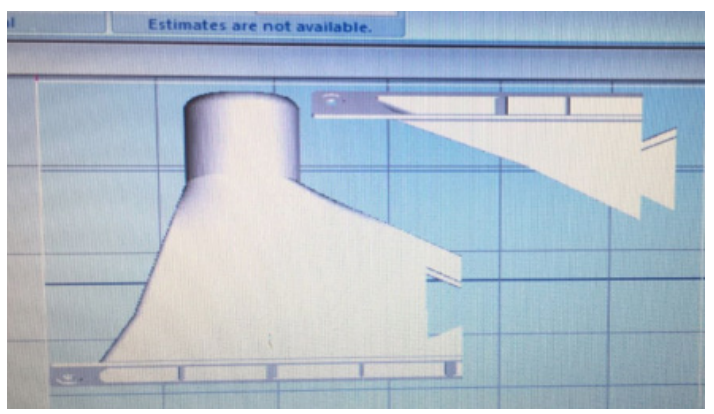


Figura 21. Impresión por partes del ducto de aire

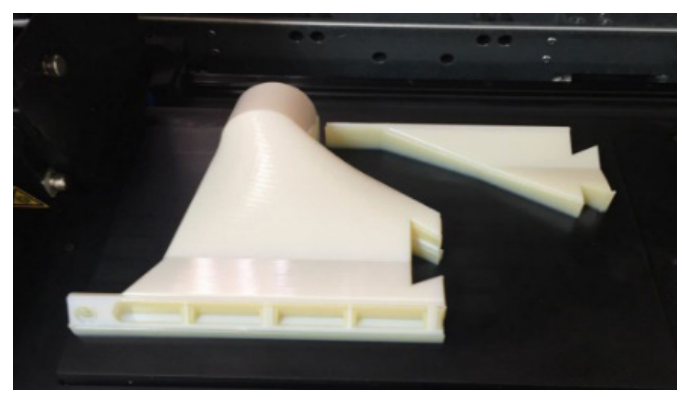

Figura 22. Ducto de Aire Solidificado

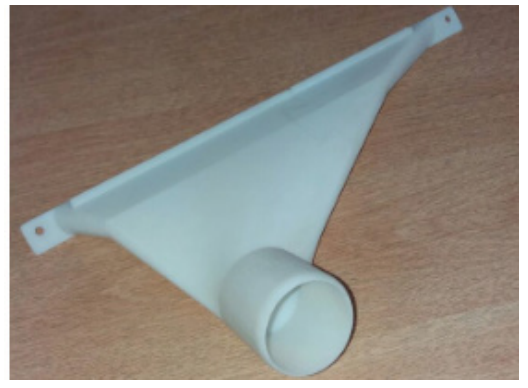

Figura 23. Impresora 3D Objet30 Pro

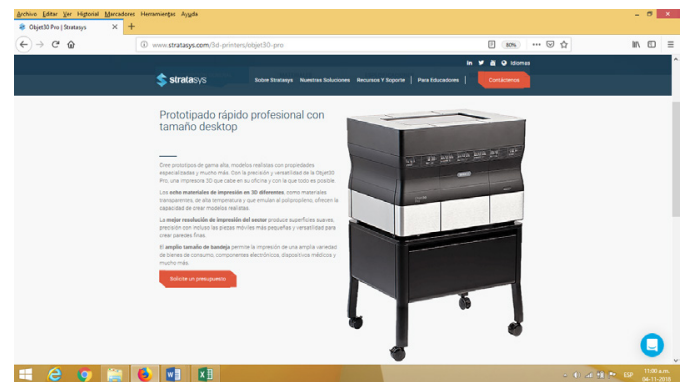

\section{Discusión}

Mediante los ensayos mecánicos (tracción y flexión) se caracterizan los materiales, dando como resultado que el material VeroWhitePlus RDG835 posee una elevada resistencia última (Su) de tracción de $55 \mathrm{MPa}$, en comparación a la del material ASA con $32 \mathrm{MPa}$ y el polipropileno con 35 $\mathrm{MPa}$. Otra propiedad mecánica es la resistencia a la rotura, donde el material VeroWhitePlus RDG835 posee un valor de $42 \mathrm{MPa}$, en comparación con el ASA su diferencia es superior en 8 MPa y 11 MPa con relación al polipropileno. En cuanto a la deflexión es superior el VeroWhitePlus RDG835 con $22.52 \mathrm{~mm}$ a diferencia del ASA $14 \mathrm{~mm}$ y el polipropileno de $14.76 \mathrm{~mm}$.

Según los resultados obtenidos en las diferentes simulaciones estructurales, se determina que el material VeroWhitePlus RGD835 presente en la Tecnología PolyJet posee una intensidad de esfuerzo de von Mises de 0.196 MPa y una deformación total de 0.058 mm, al comparar 
este polímero con el filamento ASA se prueba que no varía en cuanto a la intensidad de esfuerzo de von Mises, ya que registran valores similares. La deformación total en el material VeroWhitePlus RGD835 es superior con $0.262 \mathrm{~mm}$ respecto al filamento ASA.

El VeroWhitePlus RGD835, respecto al material original polipropileno, es superior con un valor mínimo de $0.00045 \mathrm{MPa}$ en la intensidad de esfuerzo de von Mises y en la deformación total de $0.072 \mathrm{~mm}$ la diferencia respecto al material original. En cuanto al filamento ASA tiene valores superiores comparados con el polipropileno con diferencias de $0.1918 \mathrm{MPa}$ y 0.00410 mm en la deformación total. En relación con lo que sostiene Morales (2006) existen deformaciones mínimas que sufren los materiales debido a esfuerzos constantes, por ello el análisis CFD determina puntos críticos dentro del ducto para su posterior fabricación.

La presión total del ducto de aire tiene como resultado una presión de 3.18 Pa al ingreso de la toma del ducto, lo que es una presión adecuada y resistente para flujos moderados según lo referenciado por Espinoza (2016). La velocidad del aire en el ducto en el eje X es de $2.30 \mathrm{~m} / \mathrm{s}$, en el eje $Y$ es de $0.38 \mathrm{~m} / \mathrm{s}$ y en el eje $Z$ de $2.80 \mathrm{~m} / \mathrm{s}$. Se puede determinar que la velocidad en $Z$ es adecuada para desempañar el parabrisas con velocidades de $10.08 \mathrm{~km} / \mathrm{h}$, lo que es un de flujo aire considerable en la entrada del ducto. En relación con lo que sostiene Espinosa (2016) donde las velocidades en los ejes $X$ y $Z$ poseen un mejor desempeño en el flujo de aire debido a su comportamiento geométrico y la presión inicial del sistema.

Al analizar los resultados de las comparaciones, ensayos mecánicos, simulaciones estructurales, velocidad y presión del ingreso de aire, se determina que el material VeroWhitePlus RGD835 posee una adecuada y mejor caracterización para ser usado como material constructivo por la tecnología PolyJet de Manufactura Aditiva en ductos de aire automotriz, coincidiendo con lo que sostiene el Centro de fabricación aditiva 3D (2018) y Xataca (2018).

\section{Conclusiones y recomendaciones}

De acuerdo a los resultados obtenidos a partir de los ensayos mecánicos realizados a los materiales poliméricos, se concluye que el VeroWhitePlus RDG835 posee una elevada resistencia a la tracción y un mayor módulo de elasticidad.

Si bien el material ASA es utilizado en diferentes estudios y aplicaciones, este se comercializa como un "terpolímero amorfo termoplástico", con una excepcional resistencia a la intemperie, pero el material VeroWhitePlus RGD835, garantiza mejores acabados debido que son homogéneos y tienen un alto desempeño en forma y funcionamiento tanto en piezas móviles como estáticas.

Mediante la caracterización mecánica integral, se concluye que, el material VeroWhitePlus RDG835 por impresión con tecnología PolyJet, posee una mayor capacidad de deformación plástica y de absorción de energía frente a diversas solicitaciones mecánicas en comparación con el Acrilonitrilo Estireno Acrilato ASA, por lo que puede ser considerado un "polímero de alta resistencia", pero por el contrario responde con una menor deformabilidad que el ASA.

El material VeroWhitePlus RGD835 posee un mejor comportamiento desde el punto de vista de la intensidad de esfuerzo de von Mises y deformación total en comparación con el Acrilonitrilo Estireno Acrilato ASA y el polipropileno; presentando adecuadas características en el proceso de impresión por el método PolyJet con acabado de calidad. 


\section{Bibliografía}

ASTM International. (2002, marzo 10). Standard Test Methods for Flexural Properties of Unreinforced and Reinforced Plastics and Electrical Insulating Materials. United States.

ASTM. (2012, marzo 1). Standard Terminology for Additive Manufacturing Technologies. F2792 - 12a. USA: ASTM. doi:10.1520/F2792-12A

ASTM International. (2014, Diciembre 5). Standard Test Method for Tensile Properties of Plastics. (D63814). United States.

Briceño Martínez, B. J. (2018). Evaluación de las tecnologías de impresión 3d, modelado de fusión por deposición (fdm) y tecnología polyjet, aplicada a la fabricación de conductos de aire de la camioneta toyota stout 2200. Tesis de Maestría, UISEK, Fac. Arquitectura e Ingeniería, Quito.

Cazón, A., Morer, P., y Matey, L. (2014). PolyJet technology for product prototyping: Tensile strength and surface roughness properties. Proceedings of the Institution of Mechanical Engineers, Part B: Journal of Engineering Manufacture, 228 (12): 1664-1675.

Centro de fabricación aditiva 3D. (2018). Producto3D.com. Recuperado de http://producto3d.com/materiales-impresion-3d/comparativa-materiales-fdm-2/

Espinoza Cabrera, D. M. (2016). Análisis de flujo de aire en el conducto de admisión y válvula de admisión de una motocicleta suzuki de 125cc mediante dinámica de fluidos computacional (Bachelor's thesis, Escuela Superior Politécnica de Chimborazo).

Francolí, J. F., y Díaz, R. B. (2014). Estado actual y perspectivas de la impresión en 3D. Artículos de economía industrial.

GIMDEPPM. (2018). Materials. (U. d. Barcelona, Editor) Recuperado de http://www.ub.edu/cmematerials/ es/content/polipropileno

Goncalves, R. (2002). Introducción al Análisis de Esfuerzos (2. ed.). Caracas, Venezuela: IGICA.

López, et al. (2017, septiembre 21). El método del diseño y de la ingeniería inversa en el desarrollo de planos de fabricación: una contribución a la enseñanza de la ingeniería mecánica. (37-44). Cuernavaca: Memorias del XXIII Congreso Internacional Anual de la SOMIM. Obtenido de http:// revistasomim.net/congreso2017

Lozada, A. F. V., y Suquillo, N. R. G. (2018). Análisis por el método de elementos finitos del comportamiento de las pastillas de freno ABS con base de acero y zinc al discretizar el elemento continuo al utilizar "software" CAE. Enfoque UTE, 9 (1): 188-203.Morales C., O. A. (2009). Estudio experimental y numérico del flujo de aire en ducto rectangular con un escalon. Ciudad de México, México: Instituto Politécnico Nacional.

Municipalidad de Cordoba. (2014, Julio 4). Ciencia y trabajo. Tecnología 3D. Obtenido de INFOSEPP: http:// infossep.gob.ar/

Paredes Salinas, J. G., Pérez Salinas, C. F., Miniguano, C., y Christian, B. (2017). Análisis de las propiedades mecánicas del compuesto de matriz poliéster reforzado con fibra de vidrio 375 y cabuya aplicado a la industria automotriz. Enfoque UTE, 8 (3): 1-15.

Soto de Vicente, J. (2014, junio 25). 3D Rev: Una posible revolución de la producción empresarial. (U. P. Comillas, Ed.) Facultad Ciencias Económicas y Empresariales, 1-58. Recuperado de https://repositorio.comillas.edu/xmlui/bitstream/handle/11531/86/TFG000020.pdf?sequence=1

Stratasys. (2018). Convertir la visión en realidad. Recuperado de http://www.stratasys.com/es/corporate

Toapanta Ramos, L. F., Bohórquez Peñafiel, G. A., Caiza Vivas, L. E., y Sarzosa, W. Q. (2018). Análisis numérico de los perfiles de velocidad de un flujo de agua a través de una tubería con reducción gradual. Enfoque UTE, 9 (3): 80-92.

Vega, W. H., Llanes-Cedeño, E. A., Molina, J. V., y Rocha-Hoyos, J. C. (2018). Revisión de las Características de Modelado y Optimización para el Diseño del Sistema de Suspensión Macpherson. Información tecnológica, 29 (6): 221-234. 
Villagomez, M. L. Q., Caracheo, L. A. A., Mondragón, G. R., y Castro, R. R. (2017). Revisión del estado del arte de la fabricación de multimateriales por medio de impresión 3D. Pistas Educativas, 39 (125).

Xataca. (2018). Estas son las tecnologías de impresión 3D que hay sobre la mesa y lo que puedes esperar de ellas. Recuperado de https://www.xataka.com/perifericos/estas-son-las tecnologias-deimpresion-3d-que-hay-sobre-la-mesa-y-lo-que-puedes-esperar-de-ellas 